Original Research

\title{
Small peptide targeting ANP32A as a novel strategy for acute myeloid leukemia therapy
}

\author{
Manman Wang ${ }^{\mathrm{a}}$, Hao Guo ${ }^{\mathrm{b}}$, Xuechun Zhang ${ }^{\mathrm{a}}$, Xiyang Wang ${ }^{\mathrm{a}}$, Hu Tao ${ }^{\mathrm{a}}$, Tan Zhang ${ }^{\mathrm{a}}$, \\ Min Peng ${ }^{c}$, Min Zhang ${ }^{\mathrm{d}}$, Zan Huang ${ }^{\mathrm{a}, *}$ \\ ${ }^{a}$ School of Life Sciences, Key Laboratory of Cell Hemostasis of Hubei Province, Wuhan University, No. 299 Bayi Road, Wuhan, Hubei 430072, PR China \\ ${ }^{\mathrm{b}}$ Medical Research Institute, Wuhan University, Wuhan, Hubei, PR China \\ ${ }^{\mathrm{c}}$ Department of Oncology, Renmin Hospital of Wuhan University, Wuhan, Hubei PR China \\ ${ }^{\mathrm{d}}$ Department of Hematology, Union Hospital of Huazhong University of Science and Technology, Wuhan, Hubei PR China
}

\section{A R T I C L E I N F O}

\section{Keywords:}

TAT-H3BP

ANP32A

histone $\mathrm{H} 3$

acetylation

AML therapy

\begin{abstract}
A B S T R A C T
Clinic therapy of acute myeloid leukemia (AML) remains unsatisfactory that urges for development of novel strategies. Recent studies identified ANP32A as a novel biomarker of unfavorable outcome of leukemia, which promoted leukemogenesis by increasing $\mathrm{H} 3$ acetylation and the expression of lipid metabolism genes. It is of great significance to investigate whether targeting ANP32A is a novel strategy for leukemia therapy. To target ANP32A, we identified a peptide that competed with ANP32A to bind to histone 3 (termed as H3-binding peptide, H3BP). Disrupting ANP32A and H3 interaction by the overexpression of H3BP-GFP fusion protein mimicked the effect of ANP32A knockdown, impaired H3 acetylation on multiple locus of target genes, reduced proliferation, and caused apoptosis in leukemia cells. Furthermore, a synthesized membrane-penetrating peptide TAT-H3BP effectively entered into leukemia cells and phenocopied such effect. In vivo, TAT-H3BP showed potent efficacy against leukemia: Intra-tumor injection of TAT-H3BP significantly reduced the volume of subcutaneous tumors in nude mice and recipient mice engrafted with TAT-H3BP-pretreated 6133/MPL W515L cells exhibited ameliorated leukemia burden and prolonged survival. Noticeably, TAT-H3BP efficiently suppressed proliferation and colony-forming unit of human primary AML cells without affecting normal cord blood cells. Our findings demonstrate that intervening the physical interaction of ANP32A with H3 impairs the oncogenicity of ANP32A and may be a promising therapeutic strategy against AML.
\end{abstract}

\section{Introduction}

Significant progress in genomic alterations, gene expression profiling, epigenetic modifications, and metabolic reprogramming help us understand the pathogenesis of acute myeloid leukemia (AML). However, roughly $40-45 \%$ overall survival (OS) after 5 years in 18-60 years adult patients and only approximately $10-20 \%$ surviving patients above the age of 60 years in current standard chemotherapy are far from satisfaction [1,2]. Although hematopoietic stem cell transplantation (HSCT) yields a high rate of curability for AML, it is not usually applicable for the majority of AML patients [3]. In addition, immunotherapy as a breaking-through therapy in hematopoietic malignancies is only effective in limited subtypes of leukemia [4]. Alternatively, many types of combinatory therapy were developed and currently investigated within clinical trials [5], which offered the promise of effective anti-leukemic activity and alleviating off-target effects in refractory/recurrent AML. In fact, the response to chemotherapy as well as long-term outcome of AML are affected by the heterogeneity of leukemia patients $[4,6]$. Hence, massive efforts are required to discover more therapeutic targets and more effective agents that pave the way for AML precision medicine in the foreseeable future [4].

Histone modifications are key factors affecting the initiation, development, and relapse/refractory of leukemia. The disorder of histone acetylation patterns is closely associated with drug resistance in leukemic cells and intervention of histone acetylation is a potential therapeutic strategy against AML [7]. However, the role and mechanism of histone acetylation in the occurrence, development, and treatment of leukemia have not been fully understood. Further understanding of histone acetylation is sure to define optimal therapeutic strategies against AML. For instance, recent work revealed ANP32A (acidic

\footnotetext{
* Corresponding author.

E-mail address: z-huang@whu.edu.cn (Z. Huang).
} 
nuclear phosphoprotein 32 family member A) as an unfavorable prognostic factor in AML patients and ANP32A promotes leukemogenesis by enhancing histone $3(\mathrm{H} 3)$ acetylation and upregulating the expression of lipid metabolism genes [8-10]. These findings attest that ANP32A is an unfavorably prognostic factor of AML and it is of great significance to explore whether targeting ANP32A is a novel strategy against AML.

ANP32A is characterized by a N-terminal leucine-rich repeats (LRR) domain and a disordered C-terminal acidic tail. ANP32A physically interacted with unmodified $\mathrm{N}$-terminal tail of $\mathrm{H} 3$ [11,12]. The N-terminal LRR domain of ANP32A may involve its interaction with $\mathrm{H} 3$ considering the high homology with ANP32B [13]. ANP32A lacking 151-180 amino acids failed to suppress $\mathrm{H} 3$ acetylation in vitro [12]. These findings suggest that this small region may be required for ANP32A function and short peptide spanning this region may intervene ANP32A function. Thus, we designed a competitive blocking strategy in this study and verified that the amino acid sequence spanning ANP32A 151-180 amino acids bound to H3 (termed as H3-binding peptide, H3BP). Both the overexpression of H3BP-GFP fusion protein or synthesized TAT-H3BP peptide competitively displaced ANP32A off H3. Furthermore, both H3BP-GFP and TAT-H3BP mimicked the effect of ANP32A knockdown in leukemia cells. Most importantly, TAT-H3BP showed potent efficacy against leukemia in human primary AML cells and two mouse models. Our work suggests that ANP32A is a potential target for leukemia therapy and disruption of ANP32A and H3 interaction through small peptide TAT-H3BP may be an effective anti-leukemia strategy.

\section{Materials and methods}

\section{Cell cultures and animals}

The leukemia cell lines (K562, HL60, THP1, 6133/MPL W515L) and HEK293T cells were obtained from the Chinese Center for Type Culture Collection (Wuhan, China) and cultured in complete 1640 RPMI medium (Gibco BRL, Grand Island, NY, USA) or Dulbecco's modified Eagle medium (DMEM) (Gibco BRL, Grand Island, NY, USA) supplemented with $10 \%$ fetal bovine serum, $1 \%$ streptomycin and penicillin. All animal studies were approved by the Animal Care and Use Committees of College of Life Sciences of Wuhan University.

Human primary cells isolation and culture

Bone marrow (BM) or peripheral blood (PB) were obtained from leukemia patients or healthy donors. Isolation of mononuclear cells (MNCs) from samples was described in Supplementary information.

\section{Quantitative RT-PCR}

Quantitative RT-PCR (qRT-PCR) analysis was performed as previously described [14]. Primer sets used for the qRT-PCR analysis were listed in Supplementary information.

\section{Western blotting and antibodies}

Western blotting was performed following a standard protocol [15]. Antibodies used in this study were listed in the Supplementary information.

\section{Pulldown assay}

HIS-tag pulldown assay was performed as previously described [16]. Briefly, whole cell lysates from THP1 cells and 293T cells overexpressed GFP or H3BP-GFP were used as the source of GFP, H3BP, or ANP32A protein. $6 \times$ HIS-tagged H3 was immobilized on Ni-NTA-Sepharose padding and then incubated with cell lysates and/or synthesized TAT orTAT-H3BP peptides. Then the beads were washed and the bound proteins were eluted and size-fractioned by SDS-PAGE for immunoblotting.

\section{Colony-forming unit assay}

Human primary cells were pretreated by peptides. Then colonyforming unit-granulocyte macrophage (CFU-GM) assay was performed using Methocult H4535 medium (STEMCELL, Technologies), following manufacturer's instructions. PB $\left(2 \times 10^{5}\right)$ or $\mathrm{BM} / \mathrm{CB}\left(5 \times 10^{4}\right)$ mononuclear cells were seeded in Methocult medium supplemented with $10 \%$ FBS, $2 \%$ penicillin-streptomycin solution (PS), IL-3, SCF, and GM-CSF (PeproTech, USA). Cell colonies were counted under a microscopy after 14-21 days.

\section{Cell proliferation and colony formation in soft agar}

Cell proliferation and colony formation in soft agar were performed as previously described [10]. Briefly, cell proliferation was measured by counting cell numbers for continuous 3-7days. For colony formation analysis, cells were mixed with pre-warmed soft agar suspension $(0.35 \%)$, and the mixtures were plated on the top of an underlay agar (1.0\%). Specifically, $1 \times 10^{3}$ inducible GFP/H3BP-GFP K562/HL60 cells were seeded in Fig. 1E,F, $4 \times 10^{3} \mathrm{~K} 562 / \mathrm{HL} 60 / \mathrm{THP} 1$ cells and $1 \times 10^{3}$ 6133 MPL/W515L cells pretreated by vehicle, TAT or TAT-H3BP peptides were seeded in Fig. 3C,D. Cells were cultured for 14-21 days and the colony numbers were counted under a microscopy.

\section{Subcutaneous tumor formation assay}

For the subcutaneous tumor formation assay, THP1 cells were subcutaneously injected into BALB/c nude mice as previously described [17]. Intra-tumor injection of PBS, TAT, or TAT-H3BP fusion protein was performed as described in the Supplementary information.

\section{Confocal laser scanning fluorescence microscopy}

TAT, H3BP or TAT-H3BP was added to exponentially growing cells for $12 \mathrm{~h}$ [18]. Cells were fixed with methanol, permeabilized with $0.05 \%$ Triton, and incubated with primary antibody (anti-H3) followed by a secondary antibody. Cell nuclei was stained with DAPI. Glass slides were observed with confocal laser scanning microscopy.

\section{Chromatin immunoprecipitation (ChIP)-qPCR assay}

ChIP-qPCR assay was performed as previously described [19]. Primer sets were listed in the Supplementary information.

\section{AMKL mouse model}

AMKL mouse model was established as previously described [20]. Briefly, C57BL mice were sub-lethally irradiated (400 rad) and engrafted with 6133/MPL W515L cells $\left(1 \times 10^{6}\right.$ live cells/mouse $)$ through tail vein injection. Details were described in Supplementary information.

\section{Statistical analysis}

All data were presented as means $\pm S$.D. Student's $t$-test (unpaired, two-tail) was used for comparison of two groups. * indicates $p<0.05$, and $* *$ for $p<0.01, * * *$ for $p<0.001$. NS indicates no significant difference. Results of cell proliferation assay, qRT-PCR, and flow cytometry were statistics of one representative experiment (triplicates) from at least three independent experiments with similar results. Statistics of immunoblotting was derived from the densitometric analysis of one representative blot from at least three independent experiments with similar results.

Detailed Materials and Methods were provided in the 
A

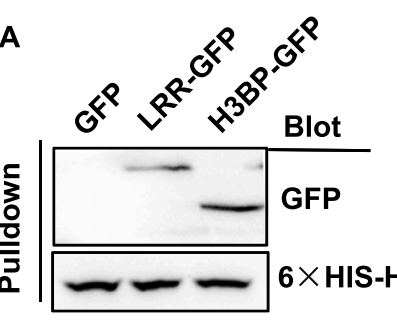

츨

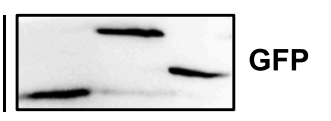

D Dox-
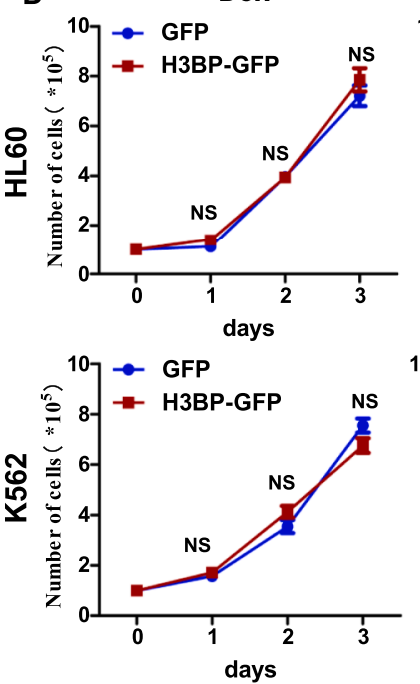

B

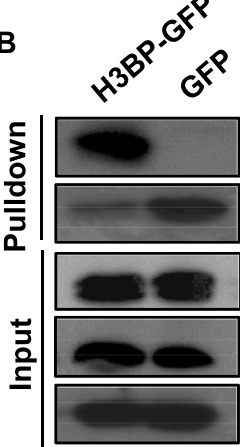

Dox+
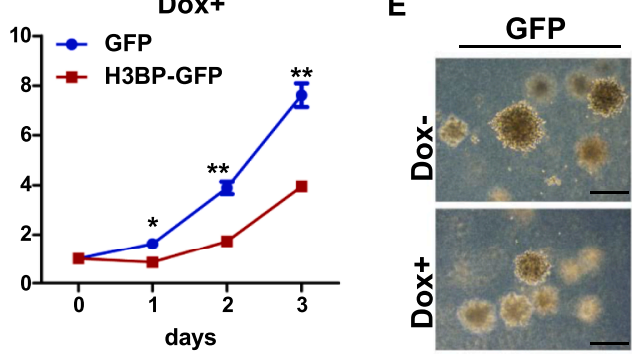

H3BP-GFP

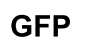
$\begin{array}{ll}\text { GFP } & \stackrel{8}{\stackrel{\Xi}{1}} \\ \text { ANP32A }\end{array}$

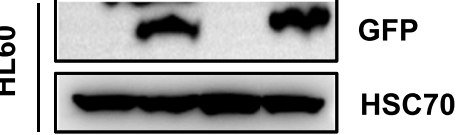

HSC70
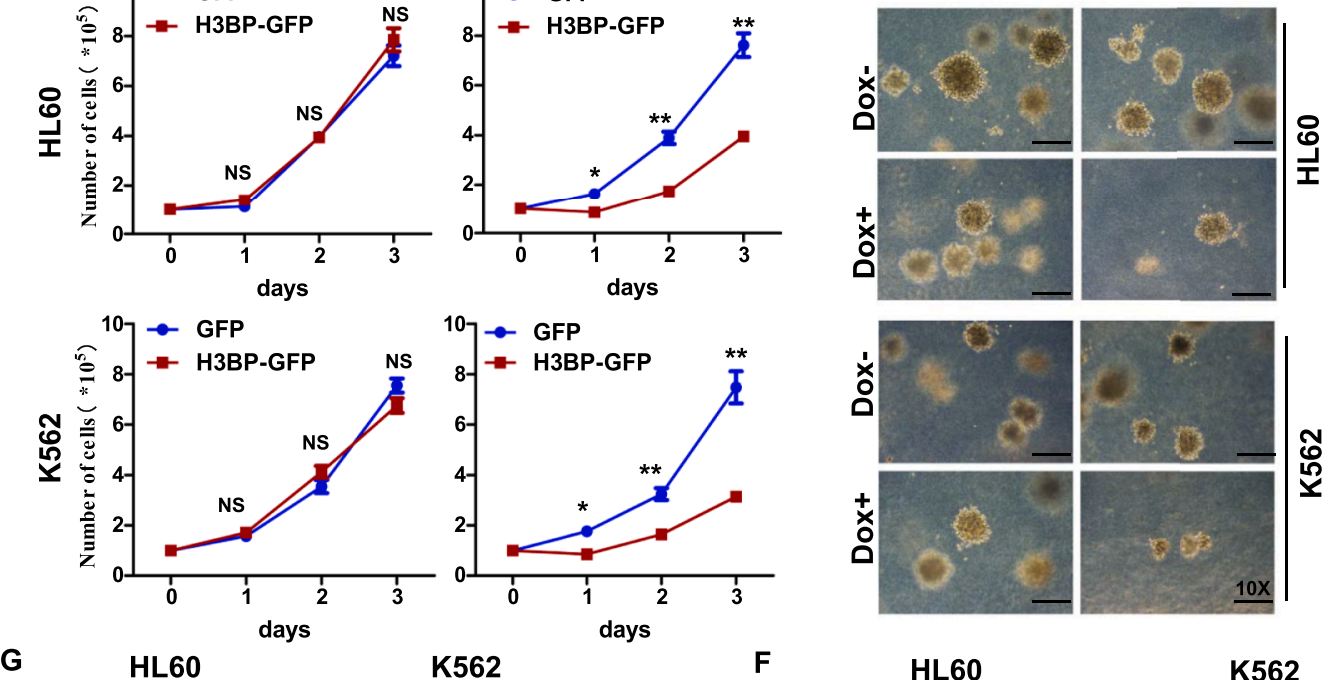

K562

\section{F}

HL60

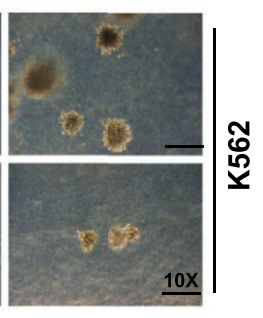

Dox $\frac{\text { GFP }}{-} \frac{\text { H3BP-GFP }}{-++} \frac{\text { GFP }}{-\quad+\frac{\text { HBP-GFP }}{-+} \text { Blot }}$
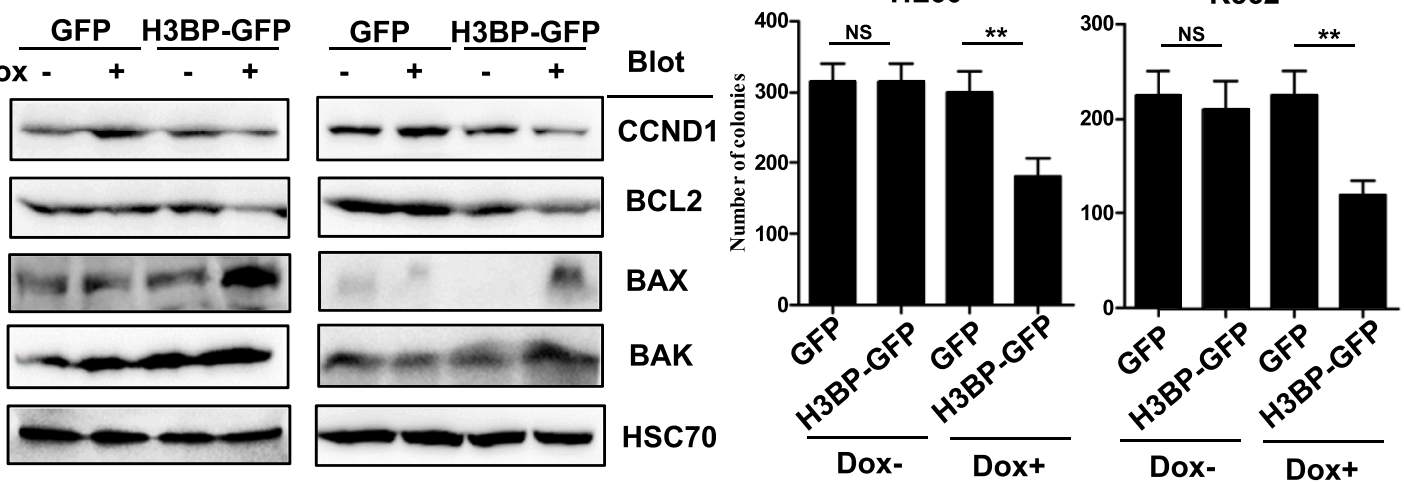

H
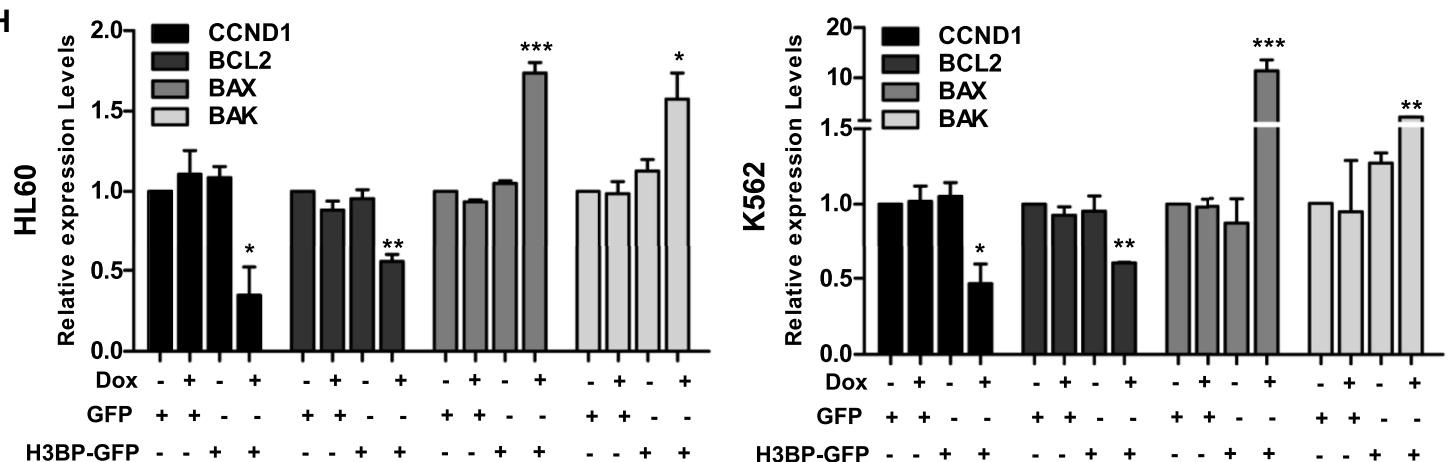

(caption on next page) 
Fig. 1. H3BP-GFP competes with ANP32A to bind to $\mathrm{H} 3$ and suppresses leukemia cell proliferation

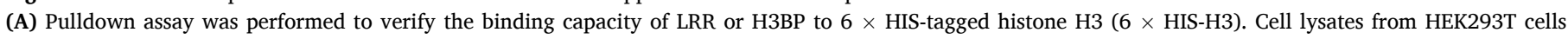

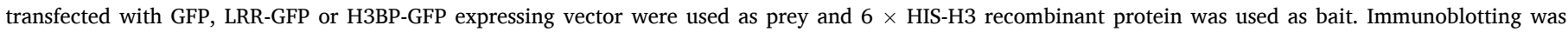

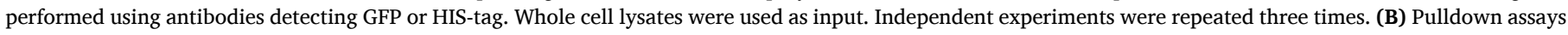

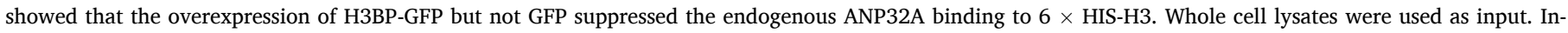

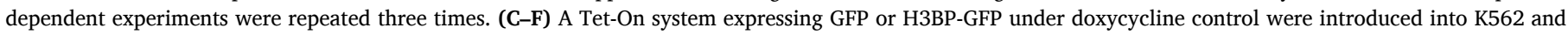

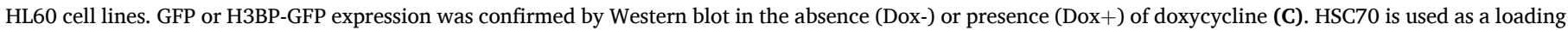

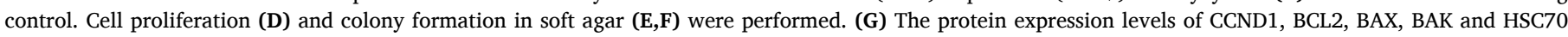

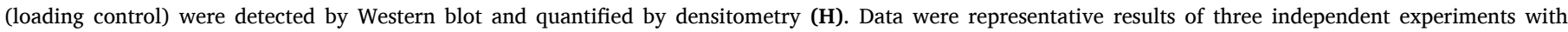
triplicates and presented as means \pm SD. $*, p<0.05 ; * *, p<0.01$ versus GFP.

\section{Supplementary information.}

\section{Results}

H3BP-GFP competes with ANP32A to bind to H3 and suppresses leukemia cell proliferation

ANP32A contributes to unfavorable outcomes for AML patients and is associated with significant $\mathrm{H} 3$ acetylation enrichment and the expression of lipid metabolism genes in leukemogenesis [8,9]. Besides, sequence spanning 151-180 amino acids of ANP32A is indispensable for its ability to suppress $\mathrm{H} 3$ acetylation in vitro $[11,12]$. It is possible that ANP32A interaction with $\mathrm{H} 3$ mediated by this sequence may be required for ANP32A function in promoting leukemogenesis. To characterize the interaction, we conducted H3 pulldown assay. The purified H3 pulled down GFP fusion with LRR domain of ANP32A (LRR-GFP) or with sequence spanning 151-180 amino acids (termed as H3-binding peptide, H3BP) of ANP32A (H3BP-GFP), but not GFP alone (Fig. 1A). Notably, H3BP-GFP overexpression effectively reduced the endogenous ANP32A binding to the exogenous H3 (Fig. 1B). To test the effect of H3BP-GFP on leukemia cell proliferation, we overexpressed GFP or H3BP-GFP under a doxycycline-inducible system. Apparently, doxycycline treatment (Dox+) efficiently induced GFP and H3BP-GFP expression in K562 and HL60 cells compared to doxycycline non-treatment (Dox-) (Fig. 1C). Consistently, the interaction of endogenous ANP32A and $\mathrm{H} 3$ was confirmed by co-immunoprecipitation and doxycycline-induced H3BP-GFP expression obstructed such interaction in K562 cell (Supplementary figure 1). GFP and H3BP-GFP cells exhibited comparable cell proliferation and colony formation in soft agar without doxycycline treatment (Fig. 1D-F). In contrast, doxycycline-induced H3BP-GFP expression significantly inhibited leukemia cell proliferation and colony formation compared to the doxycycline-treated GFP control cells (Fig. 1D-F). Moreover, H3BP-GFP impaired cell cycle and caused apoptosis evidenced by downregulation of CCND1 and BCL2 and upregulation of BAK and BAX proteins in H3BP-GFP cells treated with doxycycline (Fig. 1G,H). These results are consistent with phenotypes of ANP32A knockdown in leukemia cells in our previous study [8]. Our findings also suggest that ANP32A interaction with H3 may be indispensable for ANP32A function in leukemia. Short peptide disrupting the interaction may be an effective way to abolish ANP32A function.

\section{Synthetic Tat-H3BP impairs ANP32a interaction with $\mathrm{H} 3$ and inhibits} leukemia cell proliferation in vitro

To test whether short peptide H3BP interferes ANP32A function, we synthesized TAT-H3BP and used TAT, and H3BP for controls as previously described $[18,21]$ and labeled these peptides with FITC. K562 cells treated with TAT- and TAT-H3BP showed much higher FITC intensity than H3BP-treated cells and K562 cells treated with TAT at $30 \mu \mathrm{M}$ showed comparable FITC intensity to TAT-H3BP treatment at $80 \mu \mathrm{M}$ (Fig. 2A). Confocal laser scanning confirmed the localization of TAT and TAT-H3BP in the nuclear (DAPI and H3 staining) (Fig. 2B,C). These results suggest an efficient entry of TAT and TAT-H3BP but not H3BP into the nuclear of K562 cells. Furthermore, the capability of exogenous
H3 to pull down ANP32A overexpressed in 293T cells or endogenous ANP32A in THP1 cells was dampened by TAT-H3BP with increase of TAT-H3BP concentration compared to TAT control (Fig. 2D,E). These results suggest that the synthetic short peptide TAT-H3BP mimics H3BP-GFP to compete with ANP32A to bind to H3.

To further verify the inhibitory effect of the intracellular TAT-H3BP on leukemia cell proliferation, we pretreated K562, HL60, THP1 and 6133/MPL W515L leukemia cells with different concentrations of TATH3BP and calculated the half maximal inhibitory concentration (IC50). TAT-H3BP showed potent efficacy against leukemia cells with IC50 at micromole concentration and THP1 was the most sensitive cell line with IC50 around $40 \mu \mathrm{M}$ (Fig. 3A). Indeed, TAT-H3BP pretreatment significantly suppressed THP1 cell proliferation at $30 \mu \mathrm{M}$ and K562, HL60 and $6133 / \mathrm{MPL}$ W515L cell proliferation at $50 \mu \mathrm{M}$ over time whereas TAT or H3BP showed no significant effect (Fig. 3B). Furthermore, TAT-H3BP pretreatment of leukemia cells reduced the colony formation in soft agar (Fig. 3C,D). These cells showed reduced expression of CCND1 and BCL2 and increased expression of BAX and BAK in THP1 cells (Fig. 3E, F). These observations demonstrate that the synthetic TAT-H3BP potently disrupts ANP32A interaction with H3, suppresses leukemia cell proliferation, and causes cell apoptosis in vitro.

\section{H3BP alters $H 3$ acetylation and gene expression that mimics ANP32A knockdown in leukemia cells}

ANP32A has been shown to promote leukemogenesis by increasing $\mathrm{H} 3$ acetylation that subsequently alters the expression of lipid metabolism genes [8]. We verified that H3BP-GFP expression intervened ANP32A-mediated $\mathrm{H} 3$ acetylation and altered gene expression. Doxycycline-induced H3BP-GFP expression caused a decrease of global $\mathrm{H} 3$ acetylation (Acetyl-H3) level but not $\mathrm{H} 4$ acetylation (Acetyl-H4) (Fig. 4A,B). Particularly, ChIP-qPCR showed that H3BP-GFP expression decreased the enrichment of $\mathrm{H} 3$ acetylation on the promoters of lipid metabolism genes including APOC1, P2RX1, PCSK9, and LPPR3 compared to GFP expression (Fig. 4C). These genes as key downstream targets of ANP32A showed relatively high expression levels in AML patients compared with that in healthy donors $[8,22]$. Consistently, the expression of these genes was downregulated upon H3BP-GFP expression in comparison with the GFP expression (Fig. 4D) while no downregulation of these genes was observed without doxycycline treatment (Supplementary Fig. 2). As expected, TAT-H3BP rather than TAT peptide exerted suppressive effect on the expression of these lipid metabolism genes in leukemia cells (Fig. 4E). These results demonstrate that H3BP alters $\mathrm{H} 3$ acetylation and gene expression that mimics ANP32A downregulation in leukemia cells.

\section{TAT-H3BP exhibits potent efficacy against leukemia in vivo}

To test the in vivo function of H3BP against AML, we used two mouse models. Xenograft mouse model was established by injection of THP1 cells into BALB/c nude mice subcutaneously. When tumors were visible (around two weeks), tumor-bearing mice were randomly separated into three groups. Intra-tumor injection of PBS (Vehicle), TAT, or TAT-H3BP was performed as indicated (Fig. 5A). TAT-H3BP injection resulted in a significant decrease in tumor volume compared with Vehicle or TAT 
A

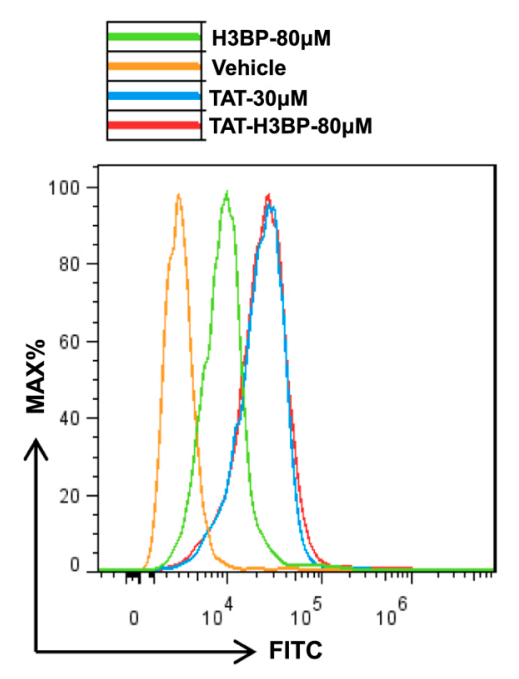

B
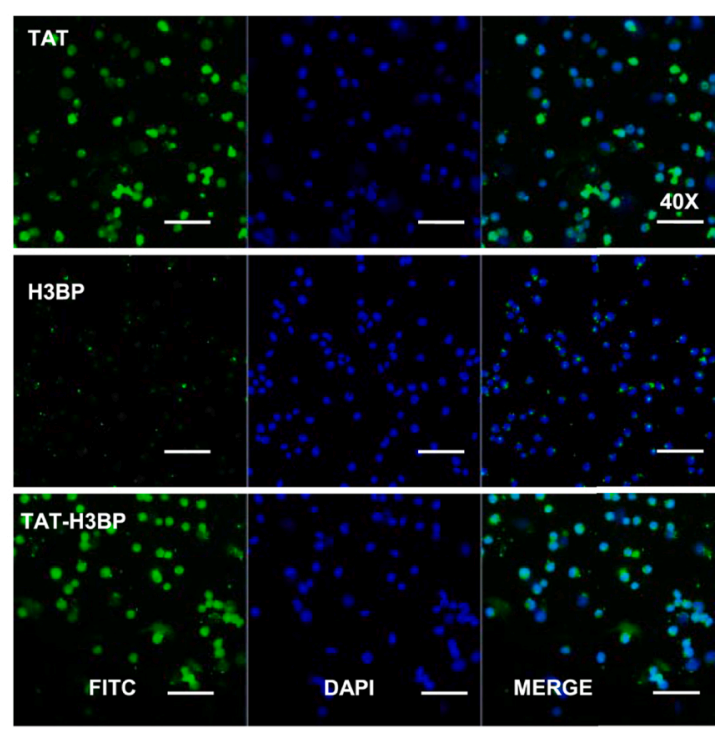

C
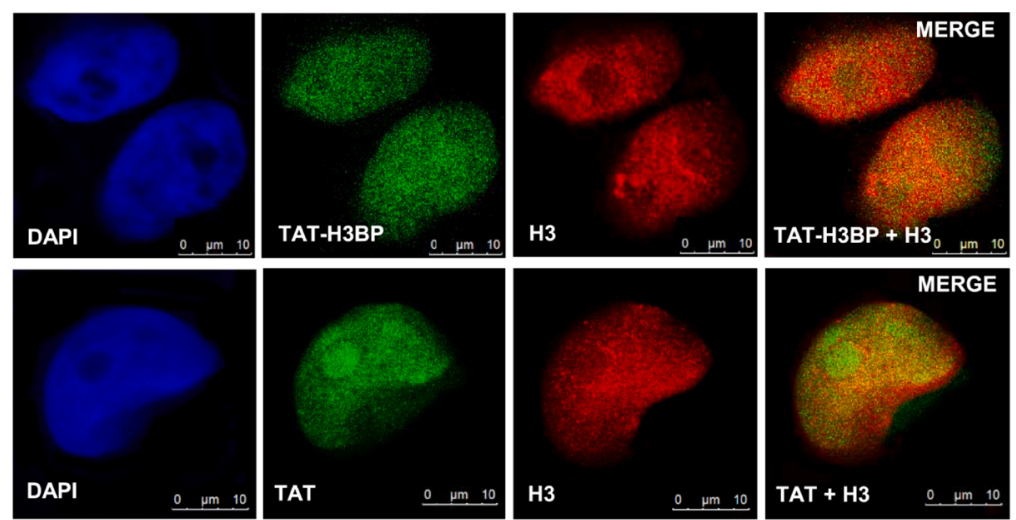

D

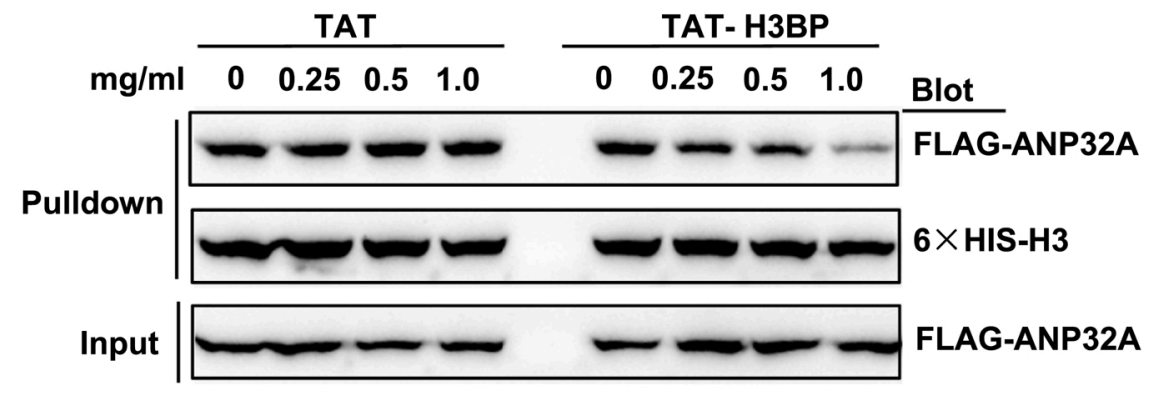

E

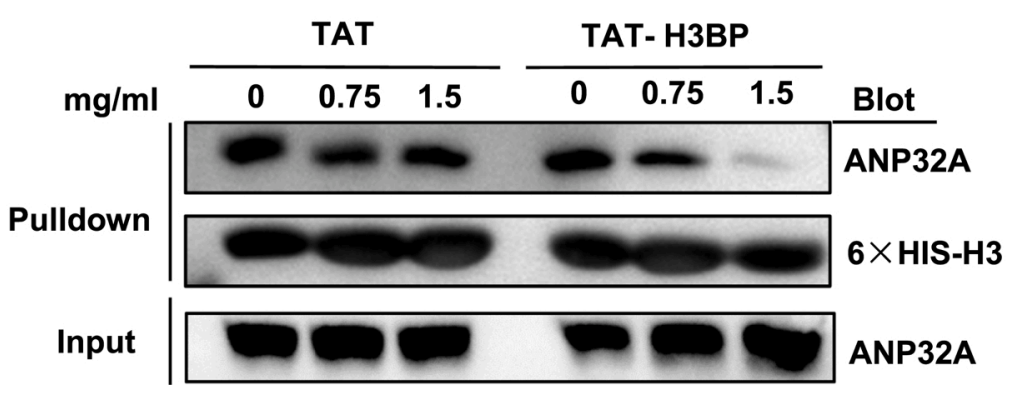




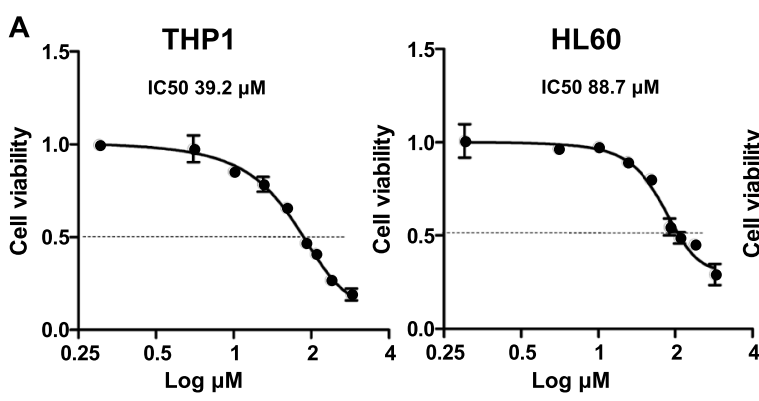

B

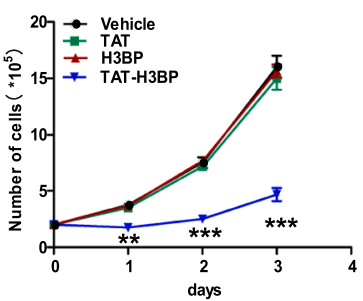

C
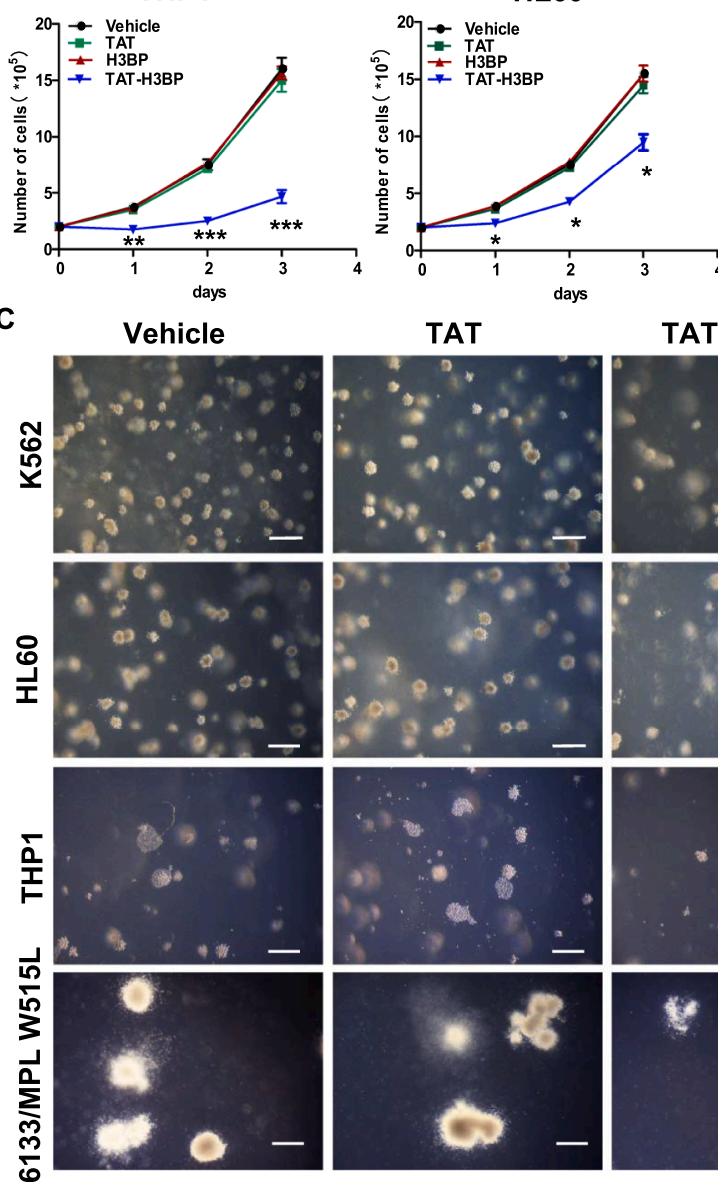

HL60

TAT
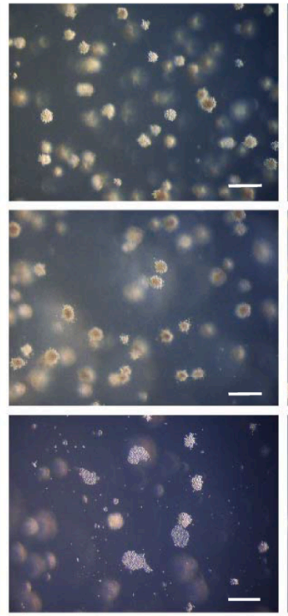

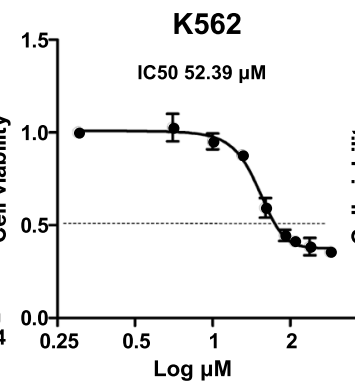

K562

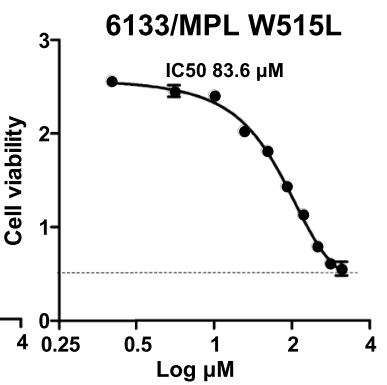

6133/MPL W515L
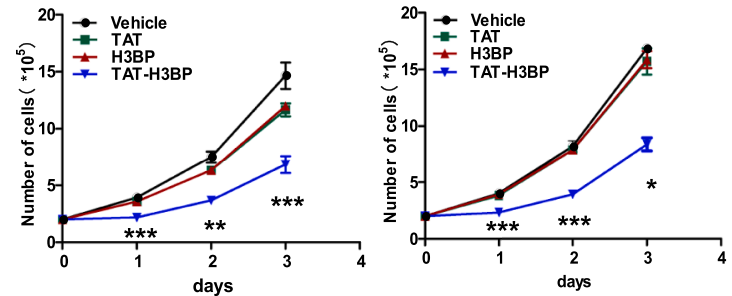

TAT-H3BP
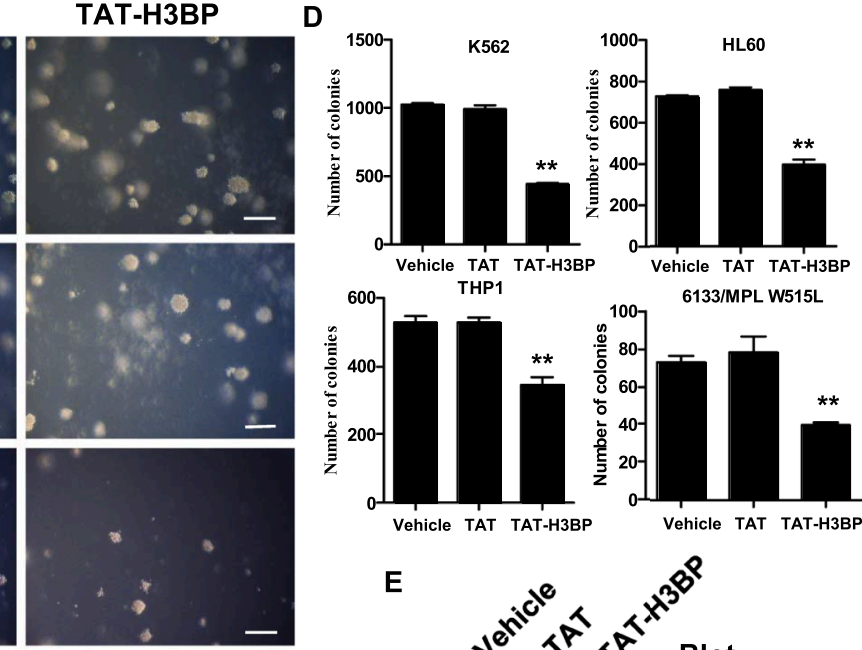

E

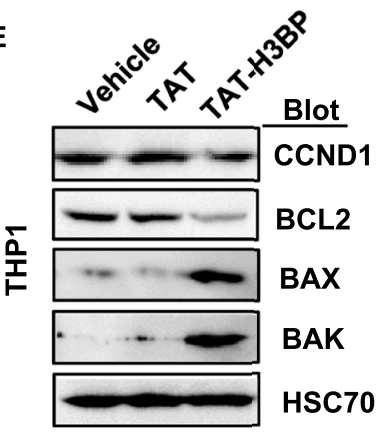

Fig. 3. TAT-H3BP inhibits leukemia cell proliferation and induces apoptosis

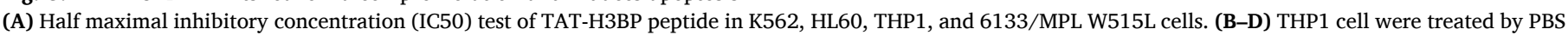

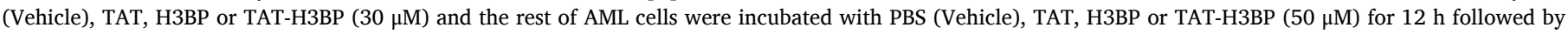

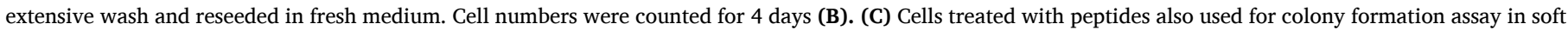

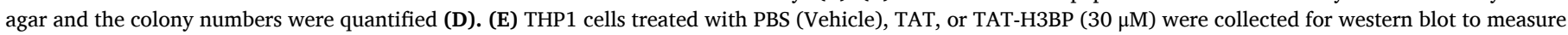

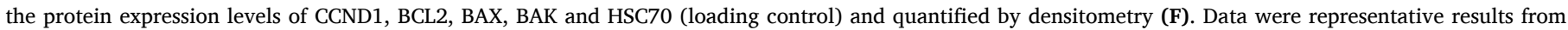

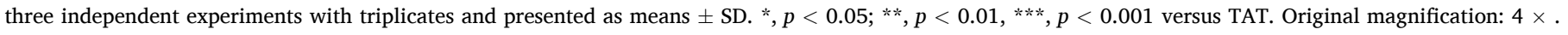


A HL60 K562

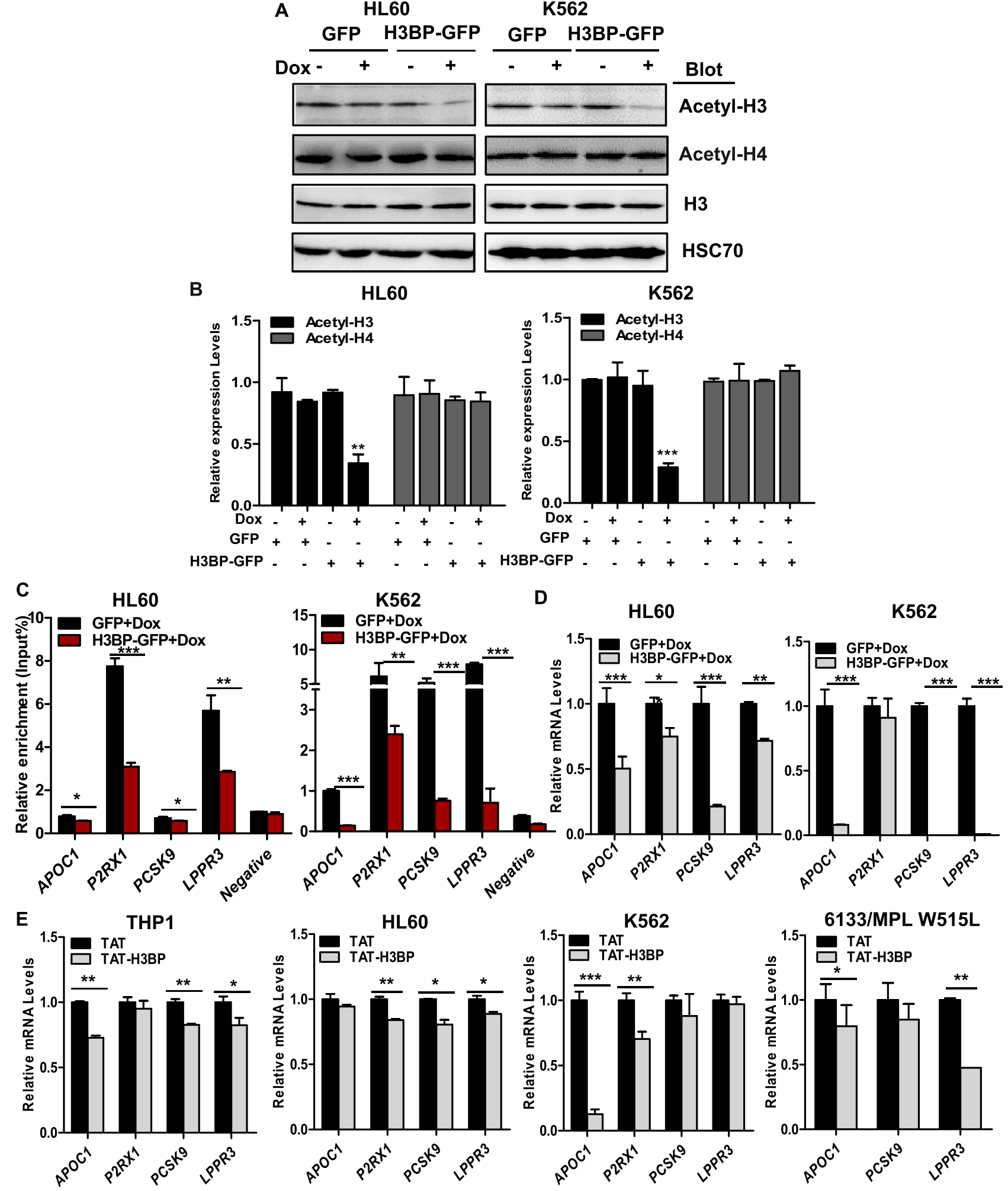

Blot

Acetyl-H3

cetyl-H4

$\mathrm{H} 3$

K562
HL60

K562

Fig. 4. H3BP alters $\mathrm{H} 3$ acetylation and gene expression that mimics ANP32A knockdown in leukemia cells

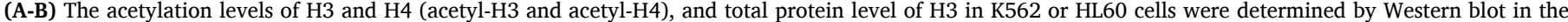

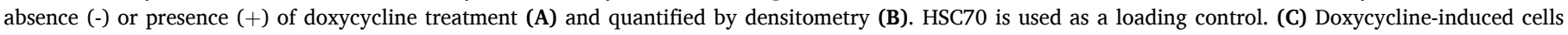

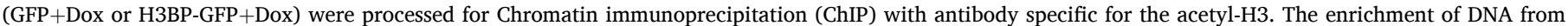

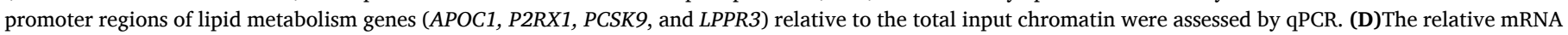

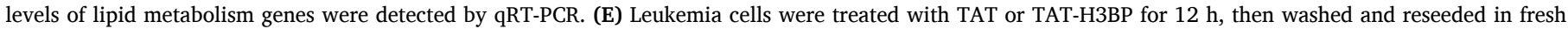

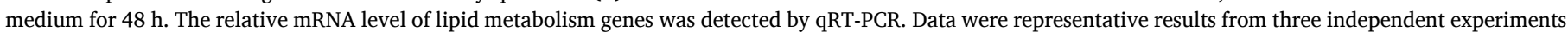
with triplicates and presented as means $\pm \mathrm{SD}$. ${ }^{* *}, p<0.01$, ${ }^{* *}, p<0.001$ versus GFP+Dox or TAT. 
A

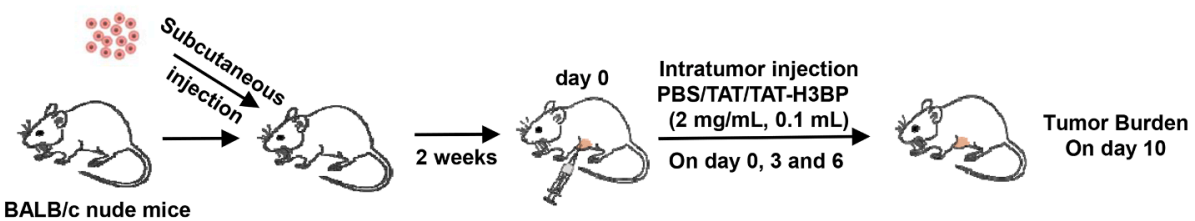

B

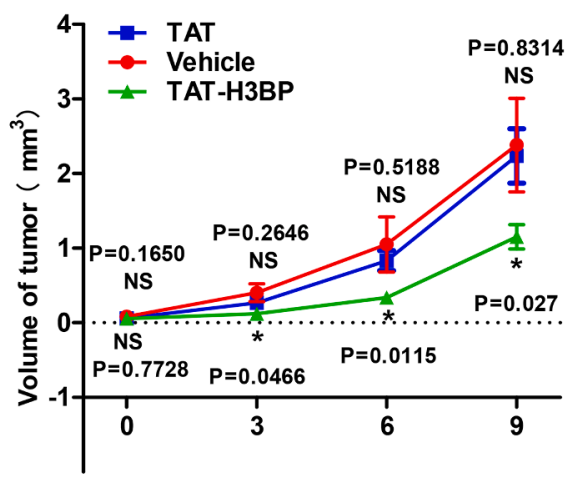

C

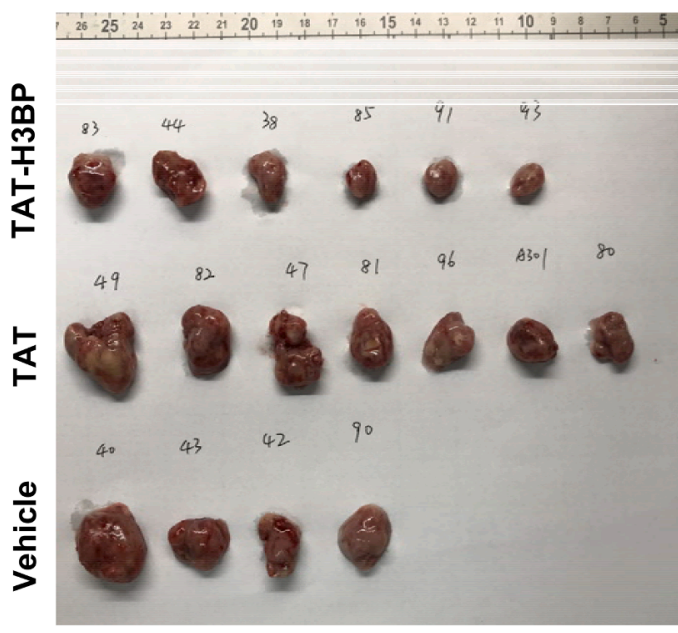

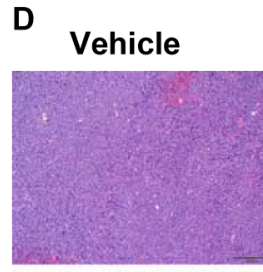

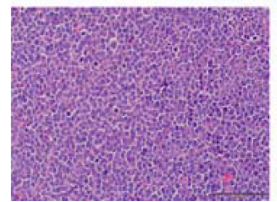

TAT
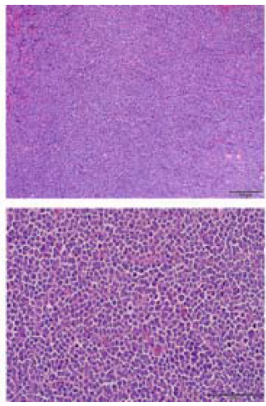

TAT-H3BP
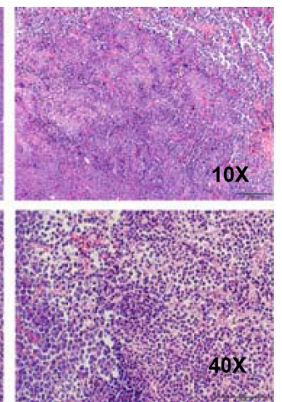

E Vehicle
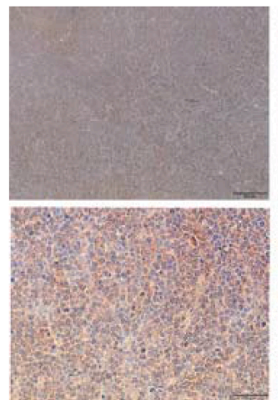

TAT-H3BP

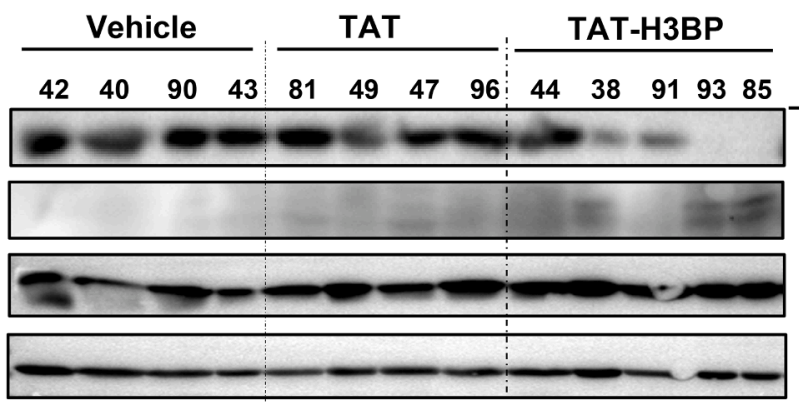

TAT
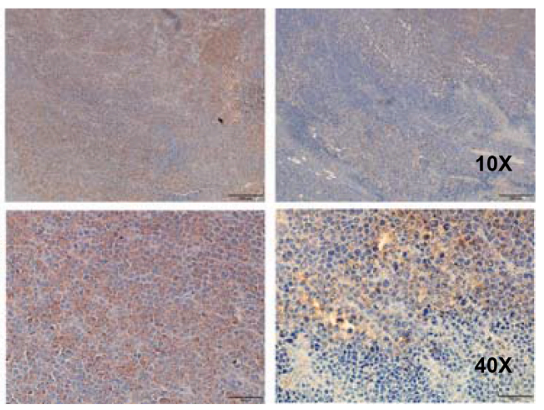

TAT-H3BP

Blot

$\mathrm{Bcl} 2$

C-Caspase 3

Bax

\section{$\beta$-Actin}

Fig. 5. TAT-H3BP impairs THP1 cell subcutaneous tumor formation in nude mice

(A) flowchart illustrating subcutaneous tumor formation of THP1 cells in nude mice and treatment with intra-tumor injection of TAT-H3BP peptide. Intra-tumor injection of PBS (Vehicle), TAT, or TAT-H3BP $(2 \mathrm{mg} / \mathrm{mL}, 0.1 \mathrm{~mL})$ were performed on day $0,3,6$ as indicated. (B) Tumor volume was recorded every 3 days. (C) Tumors were removed on day 10. (D-E) Tumor sections were stained with hematoxylin and eosin (H\&E) (D) and Ki-67 (E). Original magnification: $10 \times$ and $40 \times$. (F) The protein expression levels of Bcl2, C-Caspase3, and Bax in tumors were measured by immunoblotting. $\beta$-Actin was used as loading control.

(Fig. 5B,C). TAT-H3BP injection also augmented apoptosis and impaired cell proliferation as evidenced by increased pyknotic nuclei (shrunken and dark) and reduced Ki-67 (Fig. 5D,E). The apoptosis was also confirmed by Western blot that showed increase of cleaved Caspase 3 (C-Caspase 3), Bax, and decrease of Bcl2 (Fig. 5F).

The in vivo function of H3BP was further tested in an AMKL mouse model. 6133/MPL W515L cells have been used to induce acute megakaryocyte leukemia (AMKL) in C57BL mice $[17,20]$. We pretreated 6133/MPL W515L cells with PBS (Vehicle), TAT or TAT-H3BP for $12 \mathrm{~h}$ and intravenously injected same number of live cells into sub-lethally irradiated C57BL recipient mice (Fig. 6A). All three groups of mice showed splenomegaly and pale livers compared to irradiated control mice without injection, typical symptoms of leukemia (Supplementary Figure. 3A). Among them, TAT-H3BP pretreatment significantly delayed the onset time of AMKL and improved survival rate compared to Vehicle and TAT groups (Fig. 6B). Complete blood count (CBC) test showed a tendency of reduction in red blood cells (RBC), hemoglobin (HGB), and platelets (PLT) from Vehicle and TAT groups compared to 
A

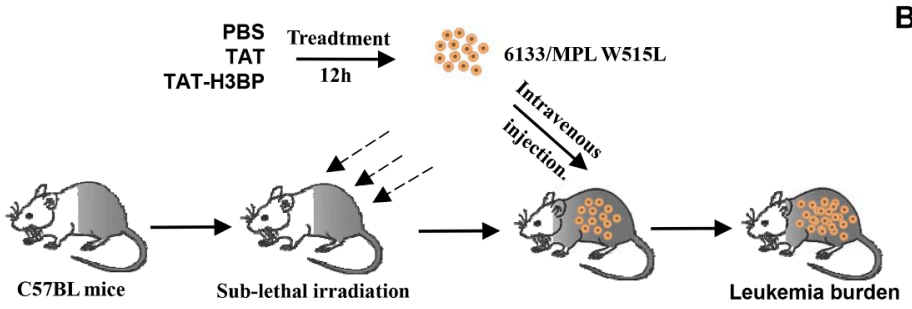

C
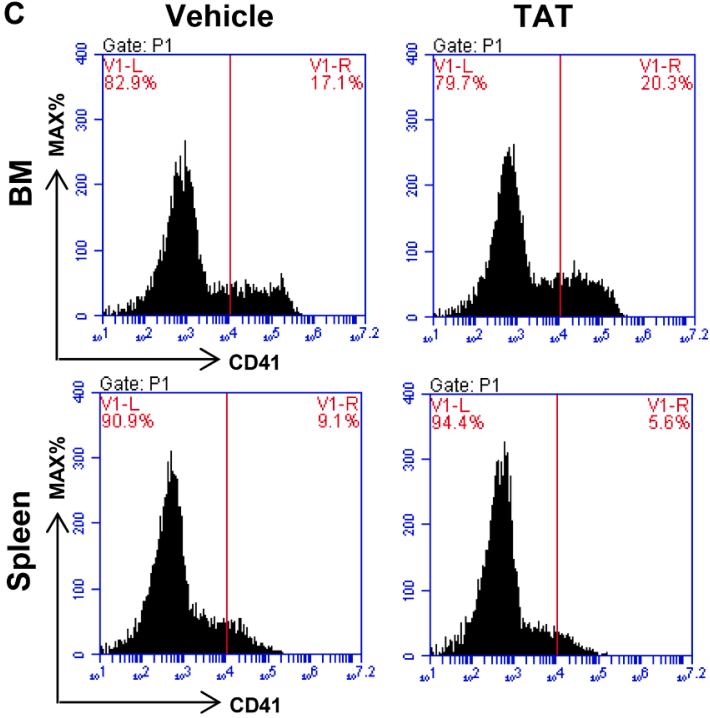

TAT
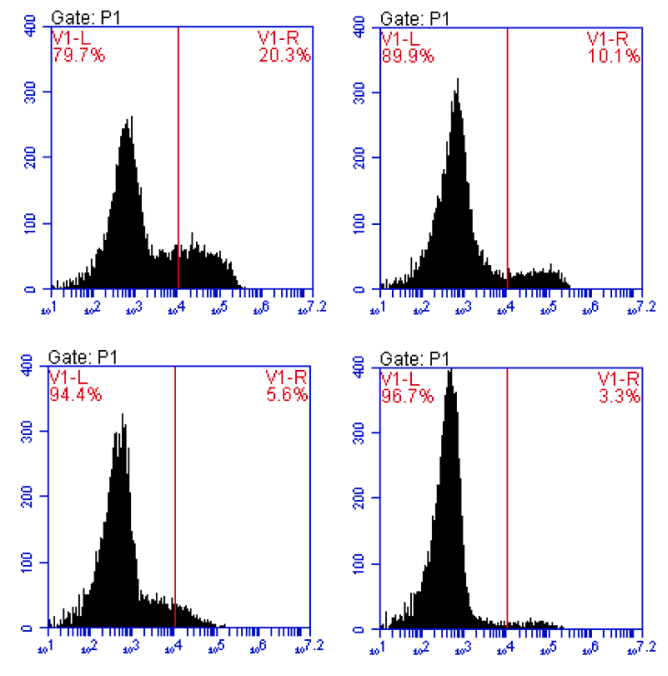

TAT-H3BP

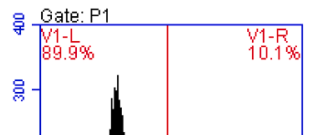

D
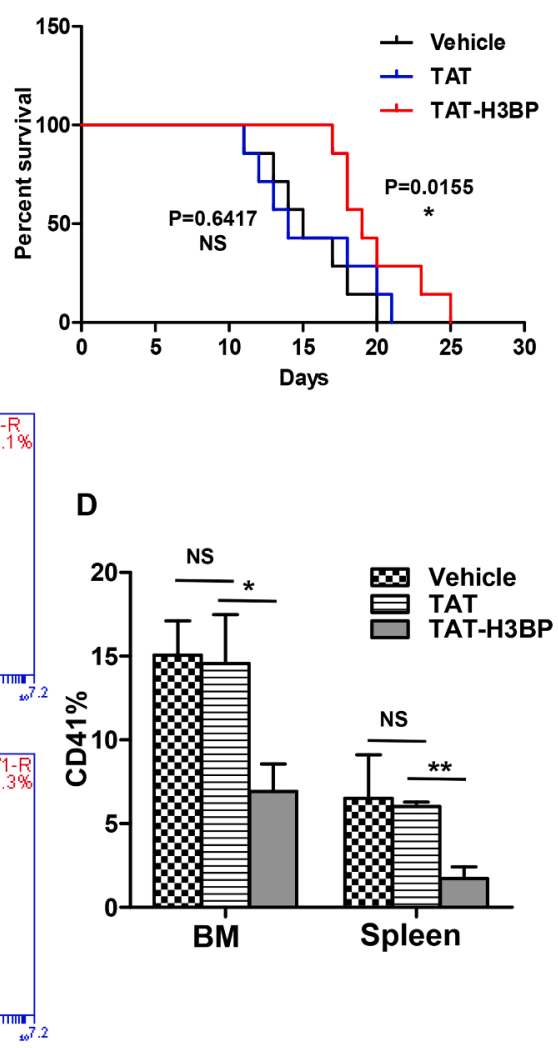

E
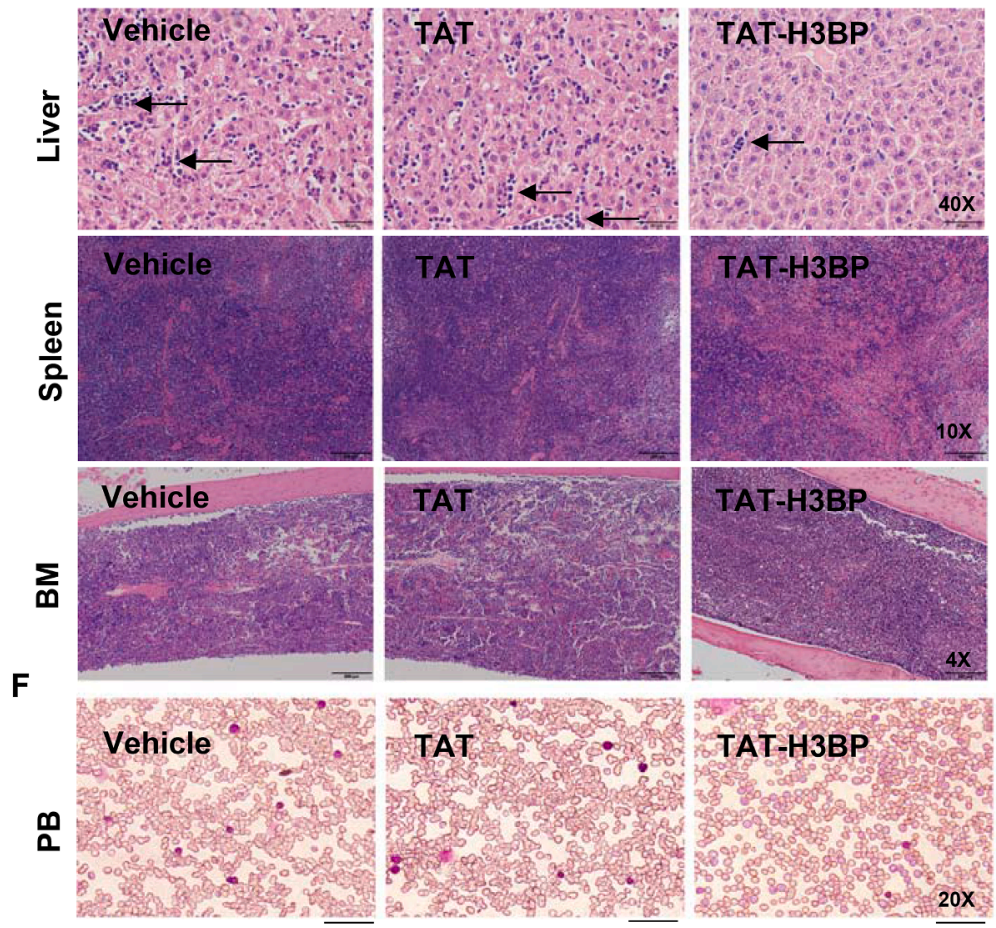
that from TAT-H3BP group (Supplementary Fig. 3B). Consistently, the percentage of $\mathrm{CD} 41{ }^{+}$cells in bone marrow (BM) and spleen from TAT-H3BP group were decreased compared with Vehicle and TAT groups (Fig. 6C,D) while the untreated control mice showed low percentage as expected (Supplementary Fig. 3C). Moreover, the pathology analysis revealed that TAT-H3BP pretreatment significantly reduced 6133/MPL W515L cell infiltration into liver and spleen, ameliorated agglomeration compared with Vehicle and TAT group (Fig. 6E). Wright's Giemsa staining showed multiplied karyocytes in peripheral blood (PB) harvested from Vehicle and TAT groups compared with TAT-H3BP group (Fig. 6F). In general, phenotypes of TAT-H3BP group mice were relatively mild in comparison with the irradiated control mice (Supplementary Fig. 3D and E). Taken together, these two mouse models confirm the in vivo function of H3BP against leukemia.

\section{TAT-H3BP dampens proliferation and survival of human primary $A M L$} cells

We further tested the efficacy of TAT-H3BP against leukemia in human primary AML cells. To exclude the non-specific effect and determine the optimal dose, we first treated mononuclear cells (MNCs) isolated from human cord blood (CB-MNC) with different concentrations of TAT, H3BP, or TAT-H3BP and perform colony-forming unit (CFU) assay. Apparently, TAT, H3BP, or TAT-H3BP did not affect CFU and the expression of lipid metabolism genes and survival genes up to $50 \mu \mathrm{M}$ (Supplementary Fig. 4A-D). At $80 \mu \mathrm{M}$ concentration, all three peptides impaired CFU (Supplementary Fig. 4A,B) indicating a nonspecific effect. Thus, we used $50 \mu \mathrm{M}$ of peptides to treat MNCs isolated from AML patient $\mathrm{PB}$ (PBMC) or bone marrow (BM) for proliferation and $\mathrm{CFU}$ assay. We confirmed the overexpression of ANP32A in many of human primary AML samples compared with normal control cells (Fig. 7A). As expected, TAT-H3BP potently impaired cell proliferation and reduced CFU (Figs. 7B-D and Supplementary 5A-D). Noticeably, TAT-H3BP-pretreated cells produced relatively smaller colonies and give rise to less cells than TAT-pretreated cells (Figs. 7E and Supplementary 5B-D). Consistently, TAT-H3BP treatment effectively suppressed the expression of ANP32A target genes including APOC1, P2RX1, PCSK9 and LPPR3 (Figs. 7F and Supplementary 6). TAT-H3BP treatment also decreased the expression of survival genes (Fig. 7G). In total, we tested the efficacy of TAT-H3BP in 11 cases of AML samples (3 BM and 8 PBMC samples) (Supplementary Table 1). Of which, cell proliferation was successfully performed in 6 cases (1 BM and 5 PBMC samples) and CFU assay in 4 cases (1 BM and 3 PBMC samples) with similar results. These observations demonstrate a potent efficacy of TATH3BP to interfere the function of ANP32A that is required for the survival of human primary AML cells.

\section{Discussion}

High expression of ANP32A contributes to AML by altering H3 acetylation on key biological processes including lipid metabolism [8]. A recent report performing multivariable analysis to evaluate the prognostic significance of ANP32A expression verified ANP32A as an unfavorable prognostic biomarker in AML risk stratification and a potential therapeutic target for AML patients [9]. In this study, we presented evidence that damaging ANP32A function by disrupting the interaction of ANP32A and H3 via H3BP was an effective strategy against AML. Both H3BP-GFP fusion and synthetic TAT-H3BP effectively interfere the interaction of ANP32A and H3 (Figs. 1A,B and 2D,E) in vitro and functionally mimicked ANP32A deficiency: H3BP-GFP fusion protein and synthetic TAT-H3BP impaired proliferation, reduced colony-forming ability, attenuated survival, decreased $\mathrm{H} 3$ acetylation, and inhibited the expression of multiple target genes in leukemia cells (Figs. 1, 3 and 4). Furthermore, TAT-H3BP exhibited potent efficacy against leukemia in vivo: Intratumor injection of TAT-H3BP peptide suppressed the subcutaneous tumor formation of THP1 cells in BALB/c nude mice and TAT-H3BP pretreatment dampened the oncogenicity of 6133/MPL W515L cells to induce AMKL in C57BL mice (Figs. 5 and 6). Most importantly, micromole concentration of TAT-H3BP peptide efficiently inhibited the proliferation and CFU of human primary AML cells (Figs. 7 and Supplementary 5-6). Our study demonstrates that intervention of ANP32A by disrupting physical interaction of ANP32A and H3 via H3BP may be an effective strategy for anti-leukemia therapy.

Our finding revealed that disruption of physical interaction of ANP32A and H3 by small molecule peptide TAT-H3BP mimics the effect of ANP32A on reducing $\mathrm{H} 3$ acetylation (Fig. 8). It is worth mentioning that the disorder of histone acetylation pattern is associated with drug resistance in leukemic cells and plays important roles in the occurrence and development of leukemia [23-25]. Although both acetylation and deacetylation have been proposed as good strategies for leukemia intervention [7], the development and clinical application of histone deacetylases inhibitors are relatively successful. For example, the pan-HDAC inhibitor Pracinostat combined with DNA methyltransferase inhibitor 5-azacytidine improved efficacy in elderly patients with AML in a phase II study (NCT01912274) [26]. In contrast, the development of histone acetyltransferase (HAT) inhibitors is impeded for lack of cell permeability or specificity, including Bisubstrate inhibitor and some natural product HAT inhibitors [27]. Alternatively, small molecule inhibitors (OTX015 [28,29], CPI-0610 and Molibresib, etc.) targeting histone acetylation readers like Bromodomain and Extra-Terminal (BET) have been tested [7]. However, their toxic effects, the mechanism of action, and true therapeutic scope remains unaddressed. Here, we present a novel strategy for intervention of histone acetylation by targeting ANP32A in AML.

Although ANP32A has been reported to be a component of acetyltransferase inhibitor in vitro, our previous study has shown ANP32A function to promote histone acetylation in AML $[8,12]$. The function of ANP32A in histone acetylation may be very dynamic and cell context depending on the cofactors recruited by ANP32A [12,30,31] (Fig. 8). ANP32A belongs to one of a family of evolutionarily conserved proteins that are characterized by an N-terminal LRR domain implicated in the mediation of protein-protein interaction [32] and a C-terminal acidic tail with a basic type of nuclear localization signal (NLS). Notably, the stronger binding of H3BP (151-180 amino acids) to H3 is critical for ANP32A regulating acetylation (Fig. 4A-C). It is possible that ANP32A, as a recruiter and subunit of histone modification and transcription complex, relies on the C-terminal acidic tail to locate in nuclear by using H3BP motif to bind to H3. It also relies on the LRR domain to recruit the cofactors such as acetyltransferases. However, TAT-H3BP peptide or H3BP-NLS-GFP protein preserves the nuclear translocation and H3 interaction but loses the "recruiter" function, which allows H3BP to interrupt endogenous ANP32A binding to H3. Theoretically, the intervention through H3BP may only specifically dampen ANP32A-mediated acetyltransferase activity but spare acetyltransferase itself. By this means, it may potentially ameliorate the toxicity of acetyltransferase inhibition by chemical compounds. Thus, our study provides a novel way to targeting histone acetylation for anti-leukemia therapy.

We also found that the effect of H3BP on cell proliferation appears to correlate to the malignancy of cells. TAT-H3BP exhibited a significantly inhibitory effect on CFU of human primary AML cells. However, TATH3BP had little effect on CFU of MNCs isolated from normal cord blood at the same micromole concentration. The addiction of leukemia cell to ANP32A may provide a therapeutic window and rationale for the clinical application of H3BP. Apart from leukemia, researches also reported that ANP32A overexpression promoted cell proliferation in various solid tumors and correlated with the progression and metastasis of cancers including colorectal cancer (CRC), hepatocellular carcinoma (HCC), oral squamous cell carcinoma (OSCC), etc. [33-35]. Our preliminary investigations also found the indispensable requirement of ANP32A in many types of cancer cells (data not shown). It is worth testing whether H3BP may exhibit anti-tumor activity in these cancers.

Our study also uncovered that the effect of TAT-H3BP seems to 
A

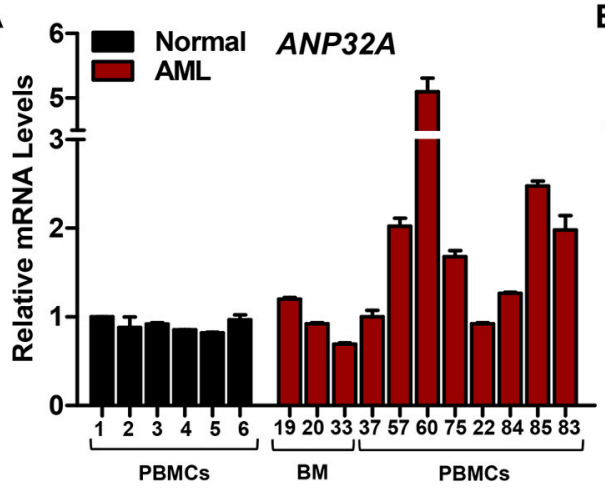

C
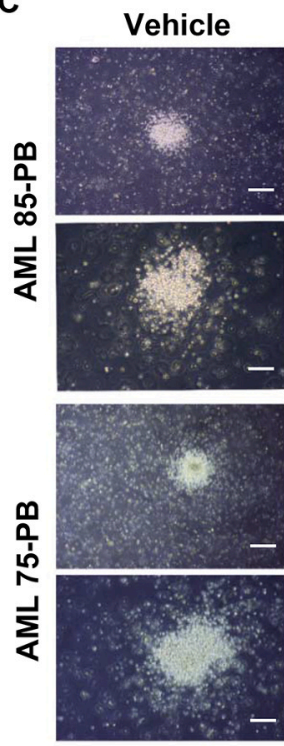

G

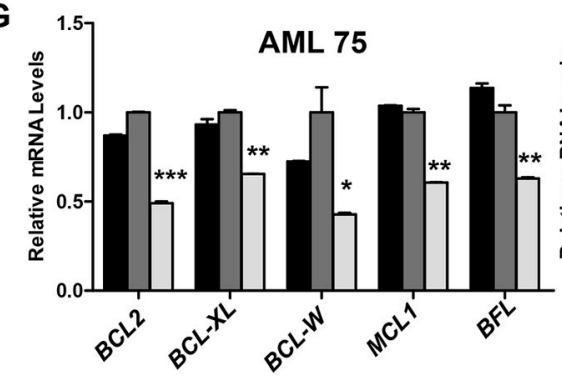

B

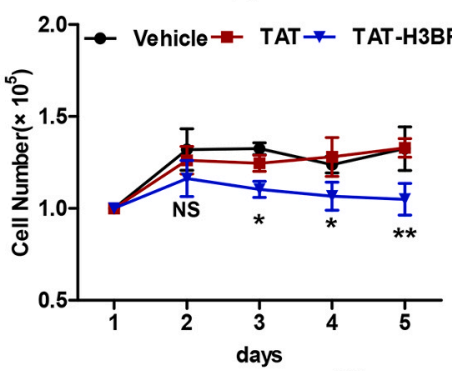

D $\quad{ }^{25} \stackrel{\text { NS }}{2 *}$

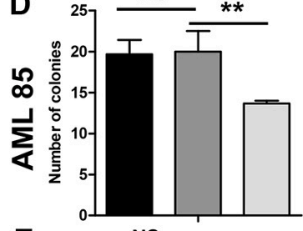

E

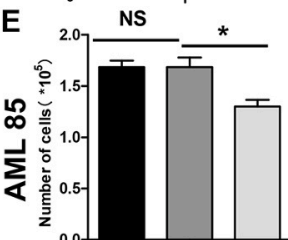

AML 19-BM
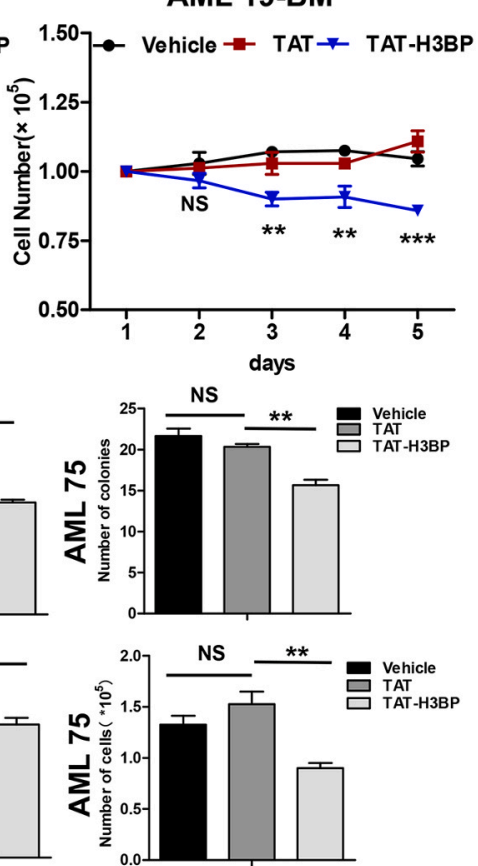

$F$
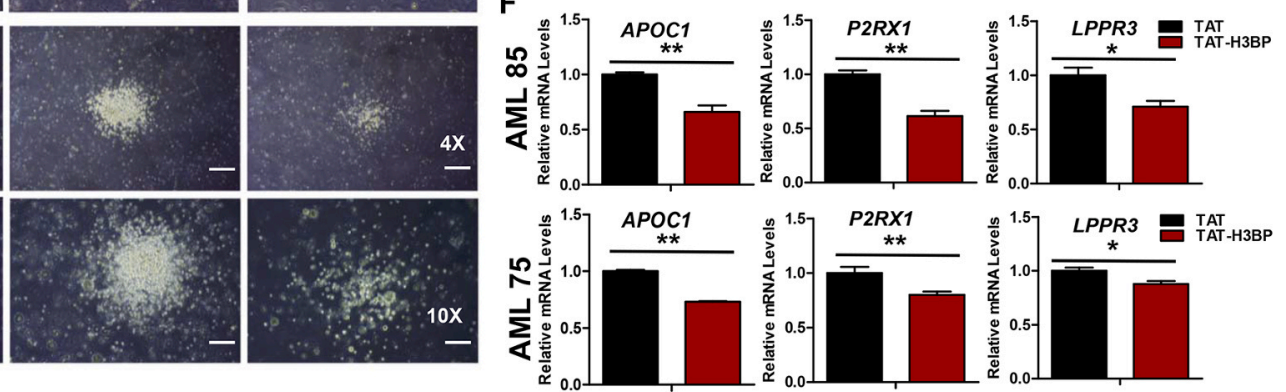

AML 60
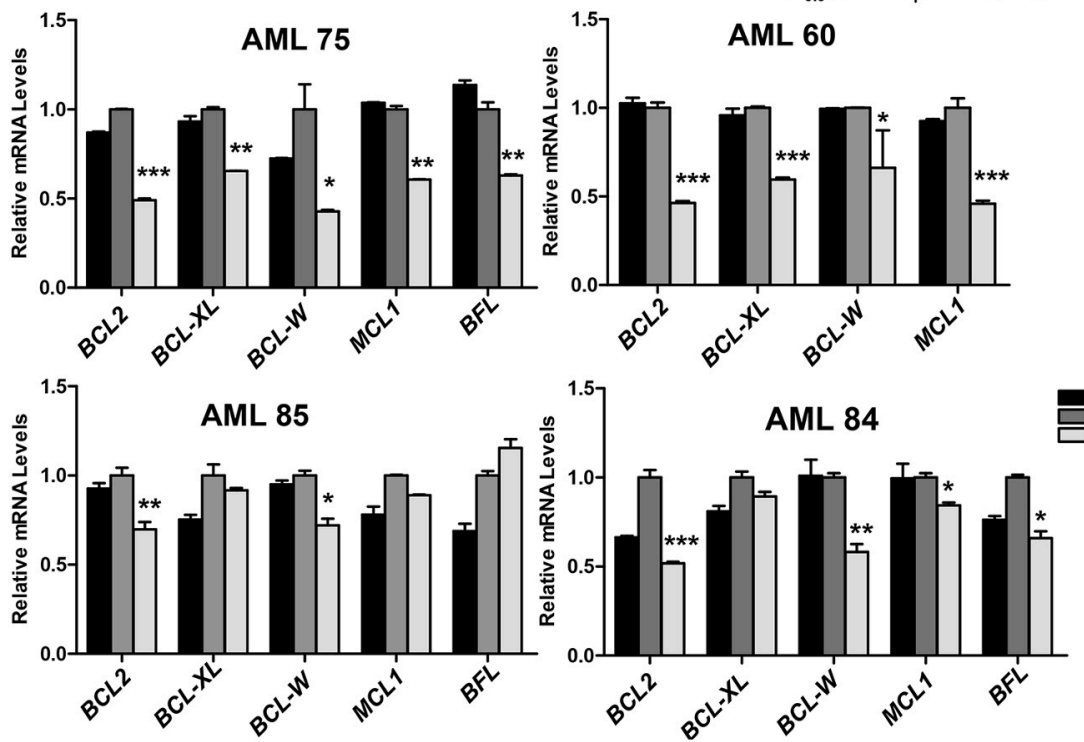

Fig. 7. TAT-H3BP dampens proliferation and survival of human primary AML cells

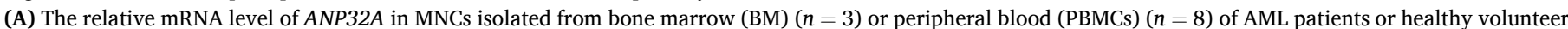

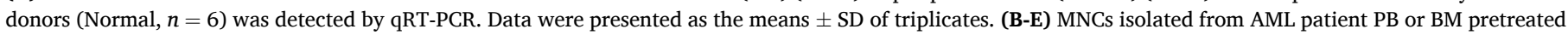

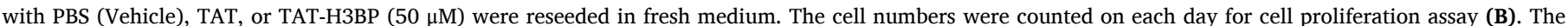

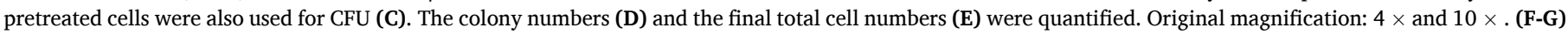

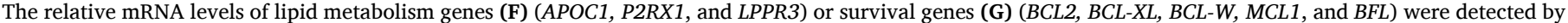
qRT-PCR in TAT or TAT-H3BP treated PBMCs. Data were presented as means \pm S.D. from triplicates. *, $p<0.05$; **, $p<0.01$, ***, $p<0.001$ versus TAT. 


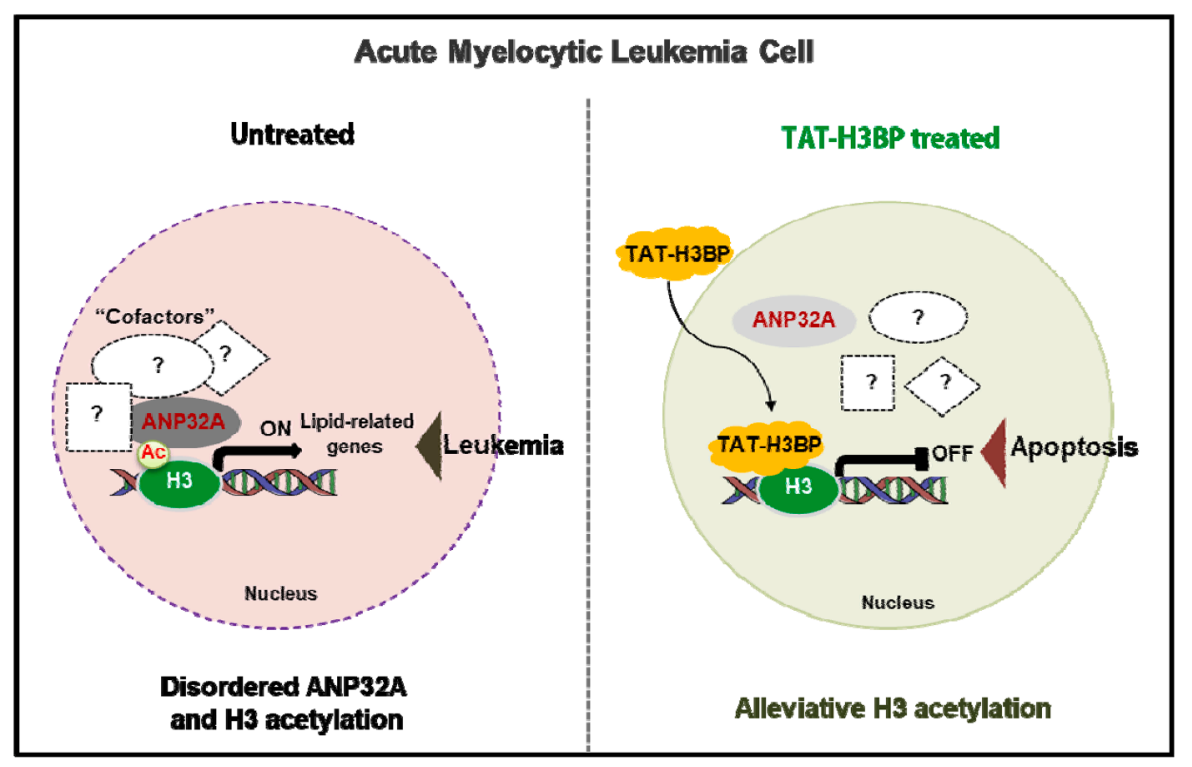

Fig. 8. Schematic illustration of H3BP targeting ANP32A as a novel strategy for leukemia therapy The abnormally high expression of ANP32A induces disordered $\mathrm{H} 3$ acetylation and gene expression of lipid metabolism genes and promotes leukemia (left). Disruption of physical interaction of ANP32A and H3 by TAT-H3BP alleviates $\mathrm{H} 3$ acetylation and the expression of lipid metabolism genes and results in cell death in leukemia cells. correlate the expression level of ANP32A. Remarkable response of PBMCs to TAT-H3BP was observed in AML patient with relatively high expression of ANP32A whereas relatively mild sensitivity was observed in AML patient with relatively low expression of ANP32A (Figs. 7 and Supplementary 5). Noticeably, TAT-H3BP showed a significant inhibition in samples from relapsed or refractory elderly patients (Fig. 7 and Supplementary Table 1) apart from newly diagnosed AML patients. Moreover, THP1 cells shows great sensitivity to H3BP treatment (Fig. 3) in consistent to that ANP32A is required for mouse bone marrow cell immortalization by MLL-AF9 [8]. Furthermore, one study indicated an overexpression of ANP32A in MLL-translocation subgroups in AML patients than normal BM [9]. Consequently, it would be very interesting to further investigate promising therapeutic potential of TAT-H3BP combined with other therapeutic agents for relapsed and refractory leukemia and to identify the subtypes of AML sensitive to TAT-H3BP therapeutic strategy. In conclusion, our findings demonstrate that targeting ANP32A as an unfavorable outcome biomarker competitively through small molecule peptide H3BP is an effective strategy against AML. It may provide scientific basis for improvement of AML targeted therapy.

\section{CRediT authorship contribution statement}

Manman Wang: Visualization, Writing - original draft, Investigation. Hao Guo: Investigation. Xuechun Zhang: Formal analysis. Xiyang Wang: Formal analysis. Hu Tao: Formal analysis. Tan Zhang: Investigation. Min Peng: Resources, Investigation. Min Zhang: Resources, Investigation. Zan Huang: Visualization, Writing - original draft, Supervision.

\section{Declaration of Competing Interest}

The authors including Manman Wang, Hao Guo, Xuechun Zhang, Xiyang Wang, Hu Tao, Tan Zhang, Min Peng, Min Zhang, and Zan Huang declare that they have no conflicts of interest in this article.

\section{Acknowledgments}

We would like to thank Drs. Hui Sun and Haining Du from College of Life Sciences Wuhan University for sharing Tet-on system and H3expressing vector, Dr. Hudan Liu from Medical Research Institute Wuhan University for mouse work, and all colleagues in our research team for technical support.

\section{Funding}

This work was supported by National Natural Science Foundation of China (91957109 and 81870427 to Z. Huang, 81770169 to M. Peng).

Ethics approval and consent to participate

All procedures involving in human samples were approved by Union Hospital of Huazhong University of Science and Technology Review Board and Renmin Hospital of Wuhan University Review Board and were consistent with the principles outlined in the Declaration of Helsinki. Consent form was obtained from each AML patient and healthy donor.

\section{Supplementary materials}

Supplementary material associated with this article can be found, in the online version, at doi:10.1016/j.tranon.2021.101245.

\section{References}

[1] J.F. Yamamoto, M.T. Goodman, Patterns of leukemia incidence in the United States by subtype and demographic characteristics, 1997-2002, Cancer Causes Control 19 (2008) 379-390.

[2] R.F. Schlenk, H. Dohner, Genomic applications in the clinic: use in treatment paradigm of acute myeloid leukemia, Hematol. Am. Soc. Hematol. Educ. Progr. 2013 (2013) 324-330.

[3] N. Khera, P. Martin, K. Edsall, A. Bonagura, L.J. Burns, M. Juckett, O. King, C. F. LeMaistre, N.S. Majhail, Patient-centered care coordination in hematopoietic cell transplantation, Blood Adv. 1 (2017) 1617-1627.

[4] X. Yang, J. Wang, Precision therapy for acute myeloid leukemia, J. Hematol. Oncol 11 (2018) 3.

[5] S. Kayser, M.J. Levis, Advances in targeted therapy for acute myeloid leukaemia, Br. J. Haematol. 180 (2018) 484-500.

[6] V. Andresen, B.T. Gjertsen, Clinical trials of repurposing medicines in acute myeloid leukemia: limitations and possibilities in the age of precision therapy, Cancer J. 25 (2019) 153-163.

[7] C.B. Benton, W. Fiskus, K.N. Bhalla, Targeting histone acetylation: readers and writers in leukemia and cancer, Cancer J. 23 (2017) 286-291.

[8] X. Yang, B. Lu, X. Sun, C. Han, C. Fu, K. Xu, M. Wang, D. Li, Z. Chen, P. Opal, et al., ANP32A regulates histone H3 acetylation and promotes leukemogenesis, Leukemia 32 (2018) 1587-1597.

[9] S. Huang, Z. Huang, C. Ma, L. Luo, Y.F. Li, Y.L. Wu, Y. Ren, C. Feng, Acidic leucinerich nuclear phosphoprotein-32A expression contributes to adverse outcome in acute myeloid leukemia, Ann. Transl. Med. 8 (2020) 345.

[10] X. Sun, B. Lu, C. Han, W. Qiu, Q. Jin, D. Li, Q. Li, Q. Yang, Q. Wen, P. Opal, et al., ANP32A dysregulation contributes to abnormal megakaryopoiesis in acute megakaryoblastic leukemia, Blood Cancer J. 7 (2017) 661. 
[11] R. Schneider, A.J. Bannister, C. Weise, T. Kouzarides, Direct binding of INHAT to H3 tails disrupted by modifications, J. Biol. Chem. 279 (2004) 23859-23862.

[12] S.B. Seo, T. Macfarlan, P. McNamara, R. Hong, Y. Mukai, S. Heo, D. Chakravarti, Regulation of histone acetylation and transcription by nuclear protein $\mathrm{pp} 32$, a subunit of the INHAT complex, J. Biol. Chem. 277 (2002) 14005-14010.

[13] B. Kobe, A.V. Kajava, The leucine-rich repeat as a protein recognition motif, Curr Opin. Struct. Biol. 11 (2001) 725-732.

[14] M. Wang, L. Tang, D. Liu, Q.L. Ying, S. Ye, The transcription factor Gbx2 induces expression of Kruppel-like factor 4 to maintain and induce naive pluripotency of embryonic stem cells, J. Biol. Chem. 292 (2017) 17121-17128.

[15] M. Wang, T. Zhang, X. Zhang, Z. Jiang, M. Peng, Z. Huang, BMP2K dysregulation promotes abnormal megakaryopoiesis in acute megakaryoblastic leukemia, Cell Biosci. 10 (2020) 57.

[16] A. Ziv-Av, N.D. Giladi, H.K. Lee, S. Cazacu, S. Finniss, C. Xiang, M.H. Pauker, M. Barda-Saad, L. Poisson, C. Brodie, RTVP-1 regulates glioma cell migration and invasion via interaction with N-WASP and hnRNPK, Oncotarget 6 (2015) 19826-19840.

[17] T. Liu, Z. Zhang, C. Yu, C. Zeng, X. Xu, G. Wu, Z. Huang, W. Li, Tetrandrine antagonizes acute megakaryoblastic leukaemia growth by forcing autophagymediated differentiation, Br. J. Pharmacol. 174 (2017) 4308-4328.

[18] Z.L. Huang, M. Gao, M.S. Ji, K. Tao, Q. Xiao, L. Zhong, J.M. Zeng, W.L. Feng, TATCC fusion protein depresses the oncogenicity of BCR-ABL in vitro and in vivo through interrupting its oligomerization, Amino Acids 44 (2013) 461-472.

[19] F.J. Yan, J. Fan, Z. Huang, J.J. Zhang, ZNF300 tight self-regulation and functioning through DNA methylation and histone acetylation, Cell Biosci. 7 (2017) 33.

[20] C. Yu, J. Zeng, Z. Yan, Z. Ma, S. Liu, Z. Huang, Baicalein antagonizes acute megakaryoblastic leukemia in vitro and in vivo by inducing cell cycle arrest, Cell Biosci. 6 (2016) 20.

[21] L. Guelen, H. Paterson, J. Gaken, M. Meyers, F. Farzaneh, M. Tavassoli, TATapoptin is efficiently delivered and induces apoptosis in cancer cells, Oncogene 23 (2004) 1153-1165.

[22] S. Zia, S. Batool, R. Shahid, Could PCSK9 be a new therapeutic target of Eugenol? In vitro and in silico evaluation of hypothesis, Med. Hypotheses 136 (2020), 109513.

[23] T. Liu, Q. Guo, H. Guo, S. Hou, J. Li, H. Wang, Quantitative analysis of histone H3 and $\mathrm{H} 4$ post-translational modifications in doxorubicin-resistant leukemia cells, Biomed. Chromatogr. 30 (2016) 638-644.
[24] M. Kahl, A. Brioli, M. Bens, F. Perner, A. Kresinsky, U. Schnetzke, A. Hinze, Y. Sbirkov, S. Stengel, G. Simonetti, et al., The acetyltransferase GCN5 maintains ATRA-resistance in non-APL AML, Leukemia 33 (2019) 2628-2639.

[25] J.E. Audia, R.M. Campbell, Histone modifications and cancer, Cold Spring Harb. Perspect. Biol. 8 (2016), a019521.

[26] J.P. Bewersdorf, R. Shallis, M. Stahl, A.M. Zeidan, Epigenetic therapy combinations in acute myeloid leukemia: what are the options? Ther. Adv. Hematol. 10 (2019), 2040620718816698.

[27] L. Simo-Riudalbas, M. Esteller, Targeting the histone orthography of cancer: drugs for writers, erasers and readers, Br. J. Pharmacol. 172 (2015) 2716-2732.

[28] E. Odore, F. Lokiec, E. Cvitkovic, M. Bekradda, P. Herait, F. Bourdel, C. Kahatt, E. Raffoux, A. Stathis, C. Thieblemont, et al., Phase I Population Pharmacokinetic Assessment of the Oral Bromodomain Inhibitor OTX015 in Patients with Haematologic Malignancies, Clin. Pharmacokinet. 55 (2016) 397-405.

[29] C. Berthon, E. Raffoux, X. Thomas, N. Vey, C. Gomez-Roca, K. Yee, D.C. Taussig, K. Rezai, C. Roumier, P. Herait, et al., Bromodomain inhibitor OTX015 in patients with acute leukaemia: a dose-escalation, phase 1 study, Lancet Haematol. 3 (2016) e186-e195.

[30] S. Kadota, K. Nagata, pp32, an INHAT component, is a transcription machinery recruiter for maximal induction of IFN-stimulated genes, J. Cell Sci. 124 (2011) 892-899.

[31] S.B. Seo, P. McNamara, S. Heo, A. Turner, W.S. Lane, D. Chakravarti, Regulation of histone acetylation and transcription by INHAT, a human cellular complex containing the set oncoprotein, Cell 104 (2001) 119-130.

[32] T.A. Santa-Coloma, Anp32e (Cpd1) and related protein phosphatase 2 inhibitors, Cerebellum 2 (2003) 310-320.

[33] B.K. Velmurugan, K.T. Yeh, C.H. Lee, S.H. Lin, M.C. Chin, S.L. Chiang, Z.H. Wang, C.H. Hua, M.H. Tsai, J.G. Chang, Y.C. Ko, Acidic leucine-rich nuclear phosphoprotein-32A (ANP32A) association with lymph node metastasis predicts poor survival in oral squamous cell carcinoma patients, Oncotarget 7 (2016) 10879-10890.

[34] W. Yan, Z. Bai, J. Wang, X. Li, B. Chi, X. Chen, ANP32A modulates cell growth by regulating p38 and Akt activity in colorectal cancer, Oncol. Rep. 38 (2017) 1605-1612.

[35] Z. Tian, Z. Liu, X. Fang, K. Cao, B. Zhang, R. Wu, X. Wen, Q. Wen, H. Shi, R. Wang, ANP32A promotes the proliferation, migration and invasion of hepatocellular carcinoma by modulating the HMGA1/STAT3 pathway, Carcinogenesis 42 (2021) 493-506. 